\title{
The Upgraded Pixel Detector of the ATLAS Experiment for Run-2 at the LHC
}

\author{
Mario P. Giordani* \\ On behalf of the ATLAS Collaboration \\ Università degli Studi di Udine \& INFN Sezione di Trieste \\ E-mail: mario.giordani@cern.ch
}

\begin{abstract}
Run-2 of the Large Hadron Collider (LHC) is providing new challenges to track and vertex reconstruction with higher energies, denser jets and higher rates. Therefore the ATLAS experiment has constructed the first 4-layer Pixel detector in HEP, installing a new Pixel layer, also called Insertable B-Layer (IBL). IBL is a fourth layer of pixel detectors, and has been installed in May 2014 at a radius of $3.3 \mathrm{~cm}$ between the existing Pixel Detector and a new smaller radius beampipe. The new detector, built to cope with high radiation and expected occupancy, is the first large scale application of 3D detectors and CMOS $130 \mathrm{~nm}$ technology. In addition the Pixel detector was refurbished with a new service quarter panel to recover about $3 \%$ of defective modules lost during run-1 and a new optical readout system to readout the data at higher speed while reducing the occupancy when running with increased luminosity. The commissioning and performance of the 4-layer Pixel Detector, in particular the IBL, will be presented using collision data.
\end{abstract}

38th International Conference on High Energy Physics

3-10 August 2016

Chicago, USA

* Speaker. 


\section{Introduction}

ATLAS is a general-purpose detector [1] on the $27 \mathrm{~km}$ Large Hadron Collider (LHC) accelerator at CERN; after Run-1, a four-year successful commissioning and operation up to $\sqrt{s}=8 \mathrm{TeV}$ and $\mathscr{L}=7 \times 10^{33} \mathrm{~cm}^{-2} \mathrm{~s}^{-1}$, at the beginning of 2013 the detector underwent the first of three long shutdown phases planned to extend the LHC physics programme into the years 2020 through dedicated upgrades. During this period a fourth layer instrumented with pixel sensors, the Insertable B-Layer (IBL), was added to the Pixel Detector [2] between a new, narrower Beryllium beampipe and the innermost Pixel Detector layer (B-Layer) [3]. At the same time, the services of the Pixel Detector were replaced by new ones (new Service Quarter Panel, or nSQP) and a new beam monitor relying on diamond and silicon 3D pixel sensors (Diamond Beam Monitor, or DBM) was installed. In Run-2, after resuming data-taking in 2015 at $\sqrt{s}=13 \mathrm{TeV}$, ATLAS has successfully operated a four-layer Pixel Detector at instantaneous luminosities surpassing the design value of $1 \times 10^{34} \mathrm{~cm}^{-2} \mathrm{~s}^{-1}$.

\section{Pixel Detector and IBL}

The Run-1 ATLAS Pixel Detector is organized in a barrel region made of three cylindrical layers and two end-caps each made of three disks on either side of the interaction point. The driving motivation for the IBL is the consolidation - and possibly the enhancement - of the tracking performance of the Inner Detector (ID) in particular in high luminosity scenarios leading to an increased number of interactions per bunch crossing (pile-up). An additional layer of highly segmented pixel sensors also helps compensating the inefficiencies caused by irreparable failures of the modules of the Pixel Detector ${ }^{1}$ and reduces the occurrence of ghost tracks induced by high pile-up backgrounds. Furthermore, the reduced distance of the IBL from the beam line (on average $3.3 \mathrm{~cm}$ ) increases the resolution on the impact parameter of the reconstructed particle tracks therefore enhancing the vertex reconstruction and b-tagging performance of the entire tracking system.
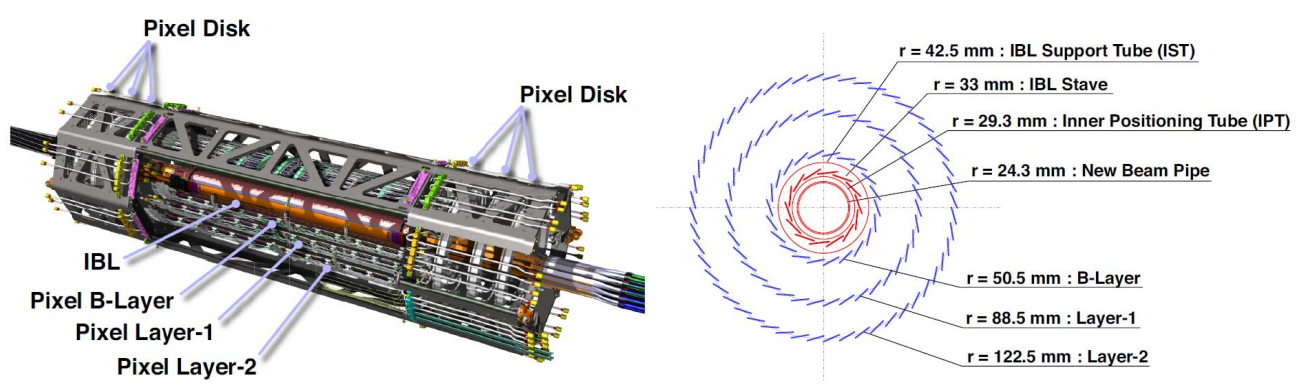

Figure 1: Schematic view of the Run-2 Pixel Detector package (left). Cross-sectional view of the Pixel Detector barrel (right); the IBL subsystem is highlighted in red.

\footnotetext{
${ }^{1}$ Irreparable failures include all issues affecting the normal operation which cannot be addressed without extracting the Pixel Detector package from ATLAS and are typically caused by radiation or by service malfunctioning. At the end of Run-1 88 pixel modules out of 1744 could not be operated any longer; most of these (55) were recovered during the first long shutdown. The occurrence of irreparable failures is expected to decrease in Run-2 thanks to the nSQP architecture, which has relocated the boards providing the electrical-to-optical signal conversion to an accessible position.
} 
As shown in figure 1, all barrel layers are arranged according to a turbine layout which maximizes the detector coverage while minimizing the charge sharing beween adjacent pixels. Due to its proximity to the interaction point, the IBL demanded a reduced pixel pitch in the beam direction $^{2}$ than the outer layers in order to cope with higher occupancies and a more radiation tolerant front-end electronics capable of higher data bandwidth which led to the development of the new FE-I4 chip [4]. Another peculiarity of the IBL is its mixed technology layout, with $75 \%$ doublechip n-in-n $200 \mu$ m-thick planar sensors [5] populating the central part of the detector and $25 \%$ single-chip n-in-p $230 \mu$ m-thick 3D devices [6] covering the high $\eta$ regions $^{3}$.

\section{Performance}

The IBL performance has been measured from a sample of $Z \rightarrow \mu \mu$ events collected in 2015 pp collisions [7]; the results are summarized in figure 2. The track-cluster association efficiency has been found to exceed $98 \%$ for particle tracks fulfilling loose quality requirements with $p_{\mathrm{T}}>0.7 \mathrm{GeV} / c$ and $|\eta|<2$; the same quantity, once projected along the beam line direction, can be used to probe the performance of the slim edge modules which caracterize the detector.
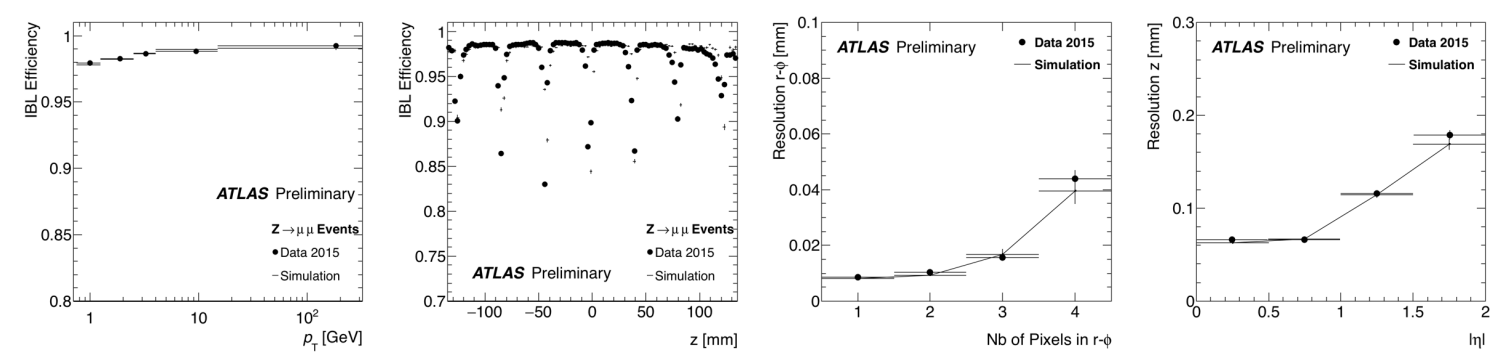

Figure 2: IBL performance [7]; from left to right: track-cluster association efficiency as a function of track $p_{\mathrm{T}}$, track-cluster association efficiency as a function of longitudinal coordinate $z$, intrinsic detector resolution in $r-\phi$ as a function of cluster size and intrinsic detector resolution in $z$ as a function of $\eta$.

A measurement of the intrinsic detector resolution has been performed on a sample of reconstructed particle tracks traversing the active region of adjacent IBL modules overlapping in $\phi$ and having two IBL hits associated to them. This method, despite being penalized in terms of statistics, does not require any extrapolation uncertainty unfolding and does not depend on track reconstruction nor on detector alignment. The detector intrinsic resolution obtained from the distribution of the distance between the two IBL clusters extrapolated to the same distance from the beam line, are found to be $(10.0 \pm 0.1) \mu \mathrm{m}$ in $r-\phi$ and $(66.5 \pm 0.8) \mu \mathrm{m}$ in $z$.

The inclusion of IBL has globally enhanced the tracking performance over the whole acceptance. Figure 3 shows this benefit in terms of impact parameter resolution by comparing two measurements performed in early Run-2 and at the end of Run-1 [8]. The improvement, which is in line with the expectations, can be explained in terms of proximity to the interaction point

\footnotetext{
${ }^{2}$ IBL pixels measure $50 \times 250 \mu \mathrm{m}^{2}$ compared to $50 \times 400 \mu \mathrm{m}^{2}$ of the outer layers.

${ }^{3} \eta=-\ln \tan \frac{\theta}{2}$, where $\theta$ is the polar angle measured from the beam $(z)$ axis; the azimuthal angle will be denoted by $\phi$.
} 
of the innermost layer (which drives the performance at low track $p_{\mathrm{T}}$ ) and smaller pixel pitch in the $z$ direction. The enhanced performance of the track reconstruction has a beneficial effect on the b-tagging techniques, which on average score a $10 \%$ increase in efficiency for similar light-jet rejection.
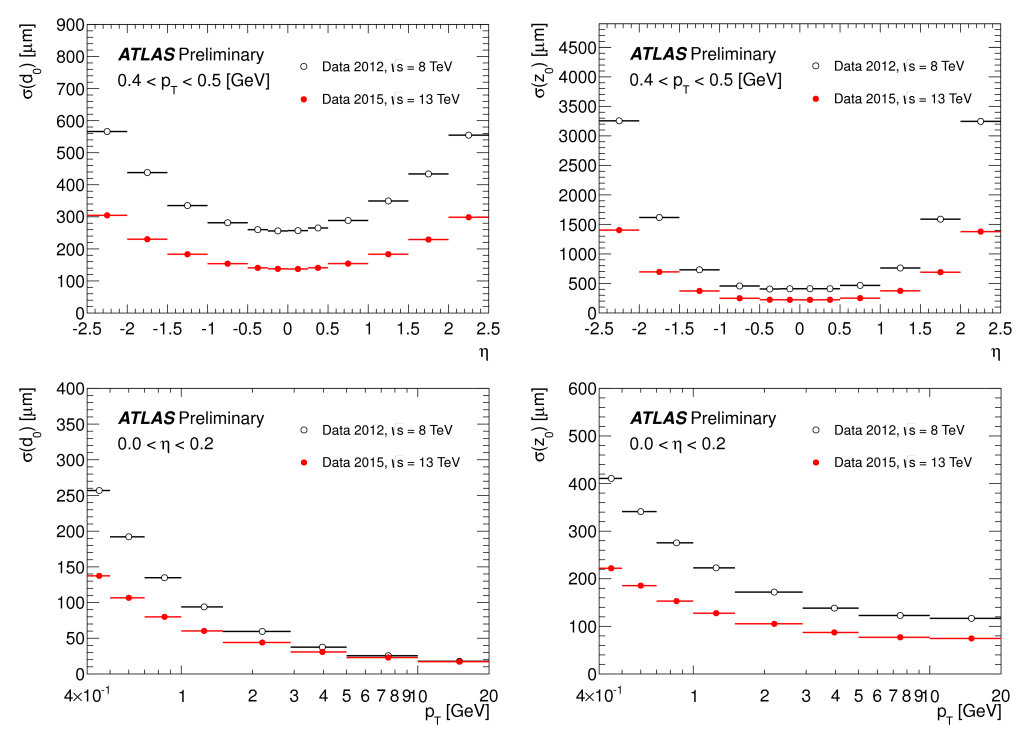

Figure 3: Comparison between 2012 Run-1 and 2015 Run-2 detector configuration [8]: transverse (left) and longitudinal (right) impact parameter resolution as functions of $\eta$ (top) and $p_{\mathrm{T}}$ (bottom).

\section{Operations}

During the first part of Run-2, the four-layer Pixel Detector has sustained data-taking at high rates (up to $85 \mathrm{kHz}$ ) with a maximum dead time of about $2 \%$; this resulted in an overall efficiency of almost 99\% during 2016. Nevertheless, rising levels of instantaneous luminosity and pile-up have required an increasing effort in finding the best compromise between data-taking efficiency and effects such as read-out desynchronization that eventually affect the track reconstruction.

Despite the good overall performance Run-2 data-taking has been influenced by a few operational issues, the most serious of which are described in more detail in the following.

\subsection{IBL Distortion}

During the first data-taking tests performed in 2015 by collecting cosmic rays, a mechanical distortion of the IBL was discovered and quickly correlated to detector temperature changes [9]. The effect, a rotational distortion around the detector axis induced by a coherent bowing of the staves (see figure 4), was eventually connected to a mismatch in the coefficient of thermal expansion between the stave carbon structure and the copper/aluminium electrical services laminated on the stave asymmetric back. The effect, quantified in $10 \mu \mathrm{m} / \mathrm{K}$ at the centre of the staves, has been confirmed by finite element analyses.

The actual position of the active components of the tracking detectors are aligned by means of a procedure that relies on the minimization of track residuals; in order to retain the tracking performance inaltered during data-taking, a time-dependent alignment procedure, the effect of which is 

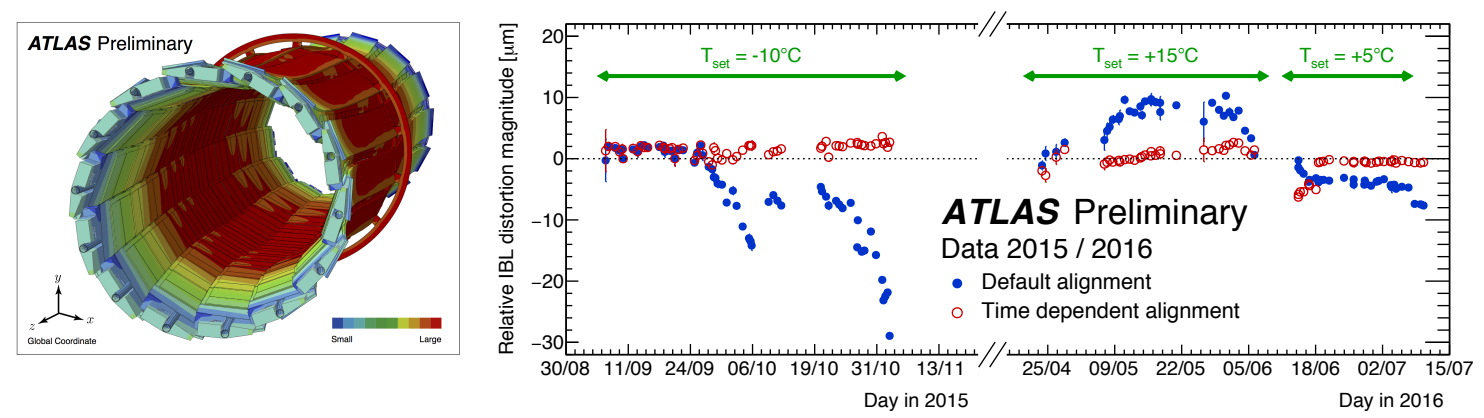

Figure 4: Left: IBL mechanical distortion reproduced by 3D finite element analysis simulation (magnified) [9]. Right: Relative bowing magnitude averaged over all IBL staves; default alignment (blue dots) are compared to the aligned geometry (red open circles) [10]. The IBL operational temperature $\left(T_{\text {set }}\right)$ for each data-taking period is also shown.

again shown in figure 4, has been enforced [10]: in this way the IBL staves can be realigned every 100 minutes.

\subsection{IBL Low Voltage Currents}

In September 2015 a steep rise in the IBL Low Voltage (LV) currents was observed [11]; figure 5 shows the magnitude of the effect, which was close to exceeding the safety limits and could lead to permanent damage in terms of module integrity, either at the wire-bond supply lines or in the FE-I4 chips in case of over-voltages induced by transient currents.

The phenomenon was correlated to the behaviour of $130 \mathrm{~nm}$ n-MOS transistors (used in the FE-I4) during early irradiation stages: hole trapping in the bulk oxide favouring the flow of a source-drain leakage current at low total ionizing dose (TID) is compensated by the generation of traps at the oxide-silicon interface, thus inducing a rebound effect. A dedicated X-ray irradiation campaign has been pursued in order to reproduce and understand this behaviour [12]; these studies unveiled different temperature dependences of the annealing processes, a fact which was eventually exploited to keep the current levels under control. For data-taking in 2016 the IBL temperature was raised to $+15^{\circ} \mathrm{C}$ to reduce the current flow and then lowered again to $+5^{\circ} \mathrm{C}$ in order to limit the detrimental effects of reverse annealing in the sensors.

\section{Towards the Future}

The effects of increasing detector occupancy are known since the end of Run-1, when the first symptoms of bandwidth limitations were observed. In order to mitigate their effects, which would otherwise become soon unsustainable in Run-2, it was decided to upgrade the off-detector read-out electronics for the two outermost pixel layers and gain a factor two in data throughput; this has been achieved by relying on the same electronics cards developed for the IBL and by exploiting the double fibres deployed with the nSQP for the Layer-1. Still under scrutiny is the upgrade of the remainig part of the detector, namely B-Layer and Disks, which - although not leading to a bandwidth increase - would benefit from a standard and more modern hardware. 

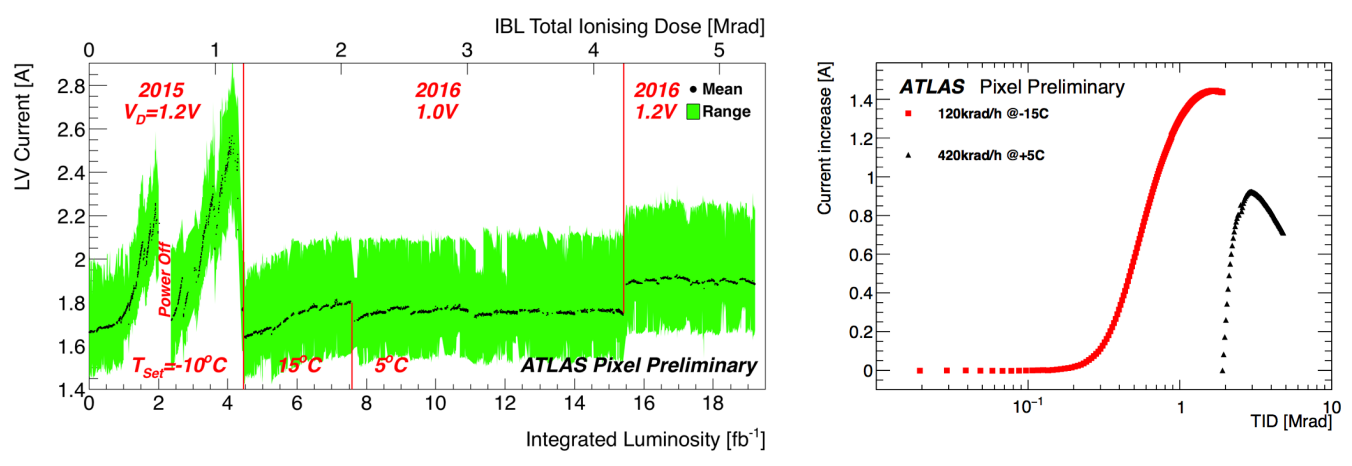

Figure 5: Left: mean Low Voltage (LV) current in IBL FE-I4 chips during stable beam as a function of integrated luminosity and corresponding total ionizing dose [11]; the changes in the supplied digital voltage $\left(V_{D}\right)$ and operational temperatures $\left(T_{s e t}\right)$ are highlighted. Right: effect of temperature and dose rate on the FE-I4 LV current as measured in dedicated X-ray campaigns [12].

\section{Conclusions}

The ATLAS four-layer 92-million pixel detector has started its operative life in 2015 and is expected to continue data-taking until the end Run-3, when the integrated luminosity will exceed by a factor ten what has been collected so far. Despite some operational issues, the detector has proven to behave reliably even beyond specifications. In order to preserve its capabilities in high luminosity scenarios, during the 2016 winter shutdown the upgrade of the off-detector electronics serving the outermost two layers will be completed.

\section{References}

[1] ATLAS Collaboration, JINST 3 S08003 (2008)

[2] G. Aad et al., JINST 3 P07007 (2008)

[3] ATLAS Collaboration, ATLAS TDR 19 (2010) and ATLAS TDR 19 ADD 1 (2012)

[4] M. Garcia-Sciveres et al., Nucl. Instrum. Meth. A 636, S155-S159 (2011)

[5] M. S. Alam et al., Nucl. Instrum. Meth. A 456, 217 (2001)

[6] S. Parker, C. Kenney and J. Segal, Nucl. Instrum. Meth. A 395, 328 (1997)

[7] “IBL Efficiency and Single Point Resolution in Collision Events"”, ATL-INDET-PUB-2016-001

[8] https://atlas.web.cern.ch/Atlas/GROUPS/PHYSICS/PLOTS/IDTR-2015-007

[9] https://atlas.web.cern.ch/Atlas/GROUPS/PHYSICS/PUBNOTES/ATL-INDET-PUB-2015-001

[10] https://atlas.web.cern.ch/Atlas/GROUPS/PHYSICS/PLOTS/IDTR-2016-005

[11] https://atlas.web.cern.ch/Atlas/GROUPS/PHYSICS/PLOTS/PIX-2016-006

[12] https://atlas.web.cern.ch/Atlas/GROUPS/PHYSICS/PLOTS/PIX-2015-008 